\title{
Una propuesta de aula invertida en la asignatura de señales y sistemas de la Universidad Nacional de Colombia
}

\author{
Ana María Reyes-Parra, Mario Julián Cañón-Ayala \& Fredy Andrés Olarte-Dussan
}

Facultad de Ingeniería, Universidad Nacional de Colombia, Bogotá, Colombia.amreyesp@unal.edu.co,mjcanona@unal.edu.co,faolarted@unal.edu.co

\begin{abstract}
Resumen - La estrategia de aula invertida ha tomado fuerza durante la última década debido a la integración de nuevas tecnologías en el ámbito educativo, facilitando el desarrollo de procesos de aprendizaje auto-regulado y de aprendizaje activo en las aulas. Teniendo en cuenta las ventajas de esta metodología, se diseñó una estrategia basada en el aula invertida para abordar el tema de "convolución" en la asignatura de Señales y Sistemas I de la Universidad Nacional de Colombia. La estrategia fue implementada con 90 estudiantes, quienes reportaron sus percepciones acerca de la estrategia propuesta a través de una encuesta. Los resultados muestran una percepción positiva en relación a la metodología y los recursos educativos propuestos. No obstante, se identificaron en algunos casos sugerencias de mejora asociadas a los recursos educativos y aspectos operativos.
\end{abstract}

Palabras Clave - Aula invertida; ingeniería; convolución; recursos educativos digitales; percepciones estudiantiles.

Recibido para revisar octubre 18 de 2017, aceptado Noviembre 16 de 2017, versión final Noviembre 23 de 2017

\section{A flipped classroom approach in the signals and systems course of the Universidad Nacional de Colombia}

\begin{abstract}
The integration of new technologies in the educational field have strengthened the development of new pedagogical strategies such as the flipped classroom, in which self-regulated and active learning are fostered. Considering the advantages of this methodology, a strategy based on the flipped classroom was designed to develop the "convolution" topic in the subject of Signals and Systems I of the Universidad Nacional de Colombia. The strategy was implemented with 90 students. After the strategy implementation, students reported their perceptions towards the flipped classroom methodology and the educational resources proposed to support the strategy development. According to the results, students had an overall positive perception in relation to the methodology and the educational resources proposed. However, suggestions for improvement of the educational resources and considerations about logistic elements, were made by some students.
\end{abstract}

Keywords_- Flipped classroom; engineer; convolution; digital educational resources; students perceptions.

\section{Introducción}

El aula invertida se considera una propuesta pedagógica útil para abordar contenidos de cursos de manera autónoma por parte de los estudiantes y aprovechar el tiempo de clase para reforzar los conceptos de manera activa a través de actividades que involucran el trabajo colaborativo y la resolución de problemas $[1,2]$. La estrategia de aula invertida ha tomado fuerza durante la última década debido a la integración de nuevas tecnologías en el ámbito educativo [3], facilitando entre otros elementos, el desarrollo de procesos de aprendizaje autoregulado a través del acceso a contenidos digitales online [2]. A diferencia de otras metodologías de enseñanza y aprendizaje online, el aula invertida incluye tiempo de interacción presencial con el docente y otros estudiantes, para discutir y aplicar los conceptos estudiados de manera autónoma por el estudiante [2].

El uso del aula invertida en ingeniería ha sido incentivado por el desarrollo de nuevas tecnologías y herramientas pedagógicas en el aula [4]. Desde el punto de vista tecnológico, los contenidos pueden ser visualizados a través de internet $\mathrm{e}$ inclusive no es necesario pagar por acceder a la información. Por ejemplo, a inicios de la década del 2000, el Instituto de Tecnología de Massachusetts (MIT) implementó la plataforma OpenCourseWare en la que actualmente se ofrecen cursos en ingeniería como el de señales y sistemas. Por otra parte, la metodología del aula invertida hace parte de la evolución de otras corrientes en las que se destaca el uso del aprendizaje activo y el aprendizaje basado en proyectos en ingeniería [4,5]. En general, la percepción de los estudiantes de ingeniería es positiva hacia el uso del aula invertida prefiriendo las actividades de aprendizaje activo en el aula y el uso de recursos de manera autónoma, aunque en ocasiones, algunos estudiantes reportan dificultades de aprendizaje ya que están acostumbrados a recibir explicaciones de parte de un docente de manera personal [4].

Durante la segunda década del siglo XXI se ha incrementado de manera significativa la implementación del aula invertida aplicada a cursos de ingeniería. Uno de los indicadores que permite evidenciar esta tendencia es la cantidad de publicaciones relacionadas con experiencias de aula invertida en ingeniería. De esta manera, se reporta que se pasaron de una a 33 publicaciones al año, entre el 2010 y el 2014 [6]. Esta metodología ha sido aplicada en cursos de ingeniería de software, cálculo, ingeniería biomédica y programación en las que se establece una estructura general con tres momentos: 1 . actividades previas a la sesión presencial utilizando recursos multimedia como videos, grabaciones,

Como citar este artículo: Reyes-Parra, A.M., Cañón-Ayala, M.J. and Olarte-Dussan, F.A., Una propuesta de aula invertida en la asignatura de señales y sistemas de la Universidad Nacional de Colombia. Educación en Ingeniería, 13(25), pp. 82-87, Febrero, 2018. 
sitios web, entre otros. 2. actividades de realimentación previas a la sesión presencial como cuestionarios y preguntas en clase. 3. Sesiones presenciales en el que se utiliza la metodología de aprendizaje activo mediante laboratorios, discusiones de problemas aplicados y teóricos, y proyectos colaborativos [79]. Dentro de estas implementaciones, la evaluación de los resultados se realiza mediante encuestas, cuyos resultados muestran que en general, los estudiantes de ingeniería prefieren esta metodología al aprendizaje centrado en el docente ya que les permite tener una mayor participación durante las sesiones grupales. Por otro lado, el aula invertida y el aprendizaje activo también han sido aplicados a cursos de señales y sistemas en cursos de pregrado y posgrado [5,10,11].

En esta línea, es importante resaltar la experiencia de Fowler en la Universidad de Binghamton quien implementó el aula invertida en estudiantes de pregrado y realizó una evaluación de acuerdo con encuestas y a la comparación de las calificaciones obtenidas en los exámenes de finales de cursos anteriores donde no se utilizaba el aula invertida [5]. La estructura general de las clases iniciaba con un vídeo que tenía por objetivo entregar una explicación de todas las temáticas a tratar más allá de hacer énfasis en la calidad visual del video. Durante la clase, Fowler aplica un cuestionario previo mediante la aplicación iClicker que es la herramienta de realimentación del trabajo realizado por los estudiantes. Finalmente, en las clases se dejan problemas a desarrollar por los estudiantes en el que algunos corresponden a aplicaciones de la teoría en códigos de ejemplo que tiene Matlab ${ }^{\circledR}$ y al final de la clase, las respuestas y explicaciones son compartidas online por el profesor y los asistentes docentes. Los resultados de esta implementación muestran una acogida de la metodología, aunque existen aspectos por mejorar en cuanto a la formación de conceptos teóricos de los estudiantes ya que el promedio de calificación obtenida en el examen final del curso con aula invertida es inferior al que se obtuvo en el año anterior sin utilizar esta metodología.

Teniendo en cuenta las ventajas que tiene el aula invertida al incorporar tecnologías de la información, posibilitar el aprendizaje autónomo y generar actividades en clase orientadas a generar una mayor apropiación de conceptos por parte de los estudiantes, se implementó esta metodología para abordar uno de los contenidos de la asignatura de Señales y Sistemas I de la Facultad de Ingeniería de la Universidad Nacional de Colombia. En las siguientes secciones de este artículo se presentan algunas generalidades de la asignatura de Señales y Sistemas I, la nueva metodología propuesta para abordar el concepto de convolución en la asignatura, las características asociadas al diseño de una encuesta para identificar la percepción de los estudiantes en relación a la nueva estrategia implementada, los resultados de aplicación de este instrumento, el análisis de las percepciones de los estudiantes y las conclusiones asociadas a la experiencia de integración de la estrategia del aula invertida en el curso Señales y Sistemas I.

\section{Metodología}

\subsection{Clase tradicional}

La asignatura de Señales y Sistemas I de la Facultad de Ingeniería de la Universidad Nacional de Colombia - Sede
Bogotá, está orientada a estudiantes de semestres intermedios y finales de los programas curriculares de Ingeniería mecánica, mecatrónica, eléctrica y electrónica. En la asignatura se aplican conceptos matemáticos para el análisis de señales y sistemas, abordando temas tales como: tipos y propiedades de señales y sistemas, caracterización de sistemas lineales e invariantes en el tiempo, análisis de señales en el dominio de la frecuencia a través de la serie y transformada de Fourier, análisis de señales y sistemas utilizando la transformada de Laplace y Z, introducción al análisis de sistemas dinámicos lineales y conceptos básicos del muestreo. Tradicionalmente, la metodología para abordar los contenidos de la asignatura integra dos tipos de sesiones: sesiones magistrales (2 horas semanales) y sesiones de taller (2 horas semanales). Durante las sesiones magistrales se presentan los conceptos y elementos teóricos asociados a una temática en particular, mientras que en las sesiones de taller, se resuelven problemas y se desarrollan ejercicios relacionados con los conceptos presentados previamente en la sesión magistral.

Pese a que esta metodología es apropiada para abordar la mayoría de temáticas y contenidos de esta asignatura, hay algunos conceptos que parecen ser difíciles de asimilar por los estudiantes a través de la presentación magistral realizada por el docente. Este es el caso particular de la temática de convolución, una operación matemática que en el marco de la presente asignatura se emplea para encontrar la respuesta o salida de un sistema (lineal e invariante en el tiempo) para una determinada señal de entrada. A lo largo de diferentes cohortes del curso se ha percibido que el tiempo de la sesión magistral es insuficiente para que los estudiantes comprendan el procedimiento analítico al aplicar la operación de convolución entre señales y en particular, para que entiendan la aplicación de este concepto en el contexto de los sistemas y las señales. Considerando estas limitaciones, se propuso implementar una estrategia de aula invertida para abordar la temática de convolución en el marco de la asignatura de Señales y Sistemas I de la Universidad Nacional de Colombia.

\subsection{Aula invertida en la asignatura Señales y Sistemas I}

La estrategia didáctica del aula invertida permite entre otros elementos aprovechar el tiempo en aula para contextualizar los conceptos teóricos que han sido revisados autónomamente de manera previa por los estudiantes. De esta manera, en el marco de la asignatura Señales y Sistemas I, se modificó la metodología de una de las sesiones magistrales integrando la estrategia de aula invertida con el fin de generar aprendizajes significativos en torno al concepto de convolución.

Con la nueva estrategia planteada, los conceptos que usualmente se presentaban durante la sesión magistral fueron abordados de manera independiente por los estudiantes a través de vídeos y otros recursos educativos digitales sugeridos por los docentes de la asignatura. Esta revisión se propuso para ser realizada por los estudiantes antes de la sesión magistral.

De esta manera, el tiempo de la sesión magistral se aprovechó para realizar actividades en las que se evidenciaba la aplicación práctica del concepto de convolución a una problemática real. En particular, se propuso a los estudiantes 
trabajar en equipo para tratar de simular la reproducción de una determinada señal de audio en diferentes recintos que habían sido caracterizados acústicamente previamente. La resolución de este problema (¿cómo suena una señal de audio en diferentes recintos?) implica la aplicación del concepto de convolución para encontrar la salida de un determinado sistema que se encuentra caracterizado por la respuesta al impulso. Durante la sesión se formulaban una serie de preguntas por parte de los docentes, que permitían orientar el trabajo de los estudiantes en el proceso de encontrar la solución a la problemática planteada. Asimismo, para esta sesión en particular se contó con el acompañamiento de docentes auxiliares que junto con el docente responsable de la magistral, interactuaban constantemente con los diferentes equipos de trabajo para resolver inquietudes y orientar a los estudiantes en la aplicación de los conceptos que enmarcaban el problema planteado.

\subsection{Identificación de percepciones de los estudiantes acerca de la nueva metodología propuesta}

Para la implementación de la estrategia de aula invertida, los docentes seleccionaron el conjunto de recursos educativos digitales (presentaciones y vídeos) para que los estudiantes los revisaran de manera autónoma. Asimismo, se prepararon los materiales de simulación necesarios para el desarrollo de la actividad práctica durante la sesión presencial.

Adicionalmente, con el fin de identificar la percepción de los estudiantes respecto a la nueva estrategia propuesta, se diseñó y aplicó una encuesta para ser diligenciada al final de la sesión presencial destinada al desarrollo de la actividad práctica. La encuesta se construyó considerando variables tales como recursos educativos propuestos (videos, presentaciones y lecturas relacionadas con el tema de convolución), metodología (estrategia del aula invertida) y aspectos operativos (tiempo disponible e instrucciones dadas para el desarrollo de actividades).

A partir de estos elementos se construyó una encuesta compuesta por 12 ítems para ser evaluados por los estudiantes a través de una escala Likert de cinco niveles: "Totalmente en desacuerdo”, “En desacuerdo”, “Ni en acuerdo o desacuerdo”, “De acuerdo” y “Totalmente en acuerdo”. En la sección final de la encuesta, se incluyeron dos preguntas abiertas para recopilar comentarios adicionales de los estudiantes sobre la metodología y recursos propuestos para abordar la temática de convolución.

\section{Resultados}

La variación metodológica propuesta para abordar la temática de convolución fue implementada con tres cursos de la asignatura Señales y Sistemas I durante el primer semestre de 2017. Los estudiantes que participaron en la implementación de esta estrategia en su mayoría eran hombres $(92,22 \%)$ y se encontraban entre el cuarto y séptimo semestre académico (82\%). De igual manera, cerca del 37\% de los estudiantes pertenecían al programa curricular de ingeniería eléctrica, el 27\% a ingeniería mecatrónica, el 25\% a ingeniería mecánica y el 11\% a ingeniería electrónica.
La encuesta propuesta para identificar las percepciones de los estudiantes sobre la nueva estrategia propuesta fue diligenciada por 90 estudiantes. Para analizar los resultados obtenidos tras la aplicación de la encuesta, se realizó un proceso de recodificación de variables para obtener un a escala numérica asociada a los diferentes niveles de la escala Likert ("Totalmente en desacuerdo"=1, "En desacuerdo"=2, "Ni en acuerdo o desacuerdo" $=3$, "De acuerdo" $=4$, “Totalmente en acuerdo"=5). De esta manera, un ítem evaluado con un valor cercano a cinco indica que el estudiante tiene una percepción positiva hacia las variables incluidas en el enunciado, mientras que un valor cercano a uno indica una percepción negativa por parte del estudiante.

En la Tabla 1 se muestran los resultados de aplicación de la encuesta de percepciones. En particular, se presentan los promedios asociados a la valoración asignada a cada ítem junto con la desviación estándar.

Los resultados presentados en la Tabla 1 indican que la mayoría de estudiantes consideraron que la actividad práctica propuesta les ayudó a entender mejor el concepto de convolución y que las actividades preparatorias -previas a la sesión presencial- fueron

Tabla 1

Promedio y desviación estándar asociada a los ítems de la encuesta

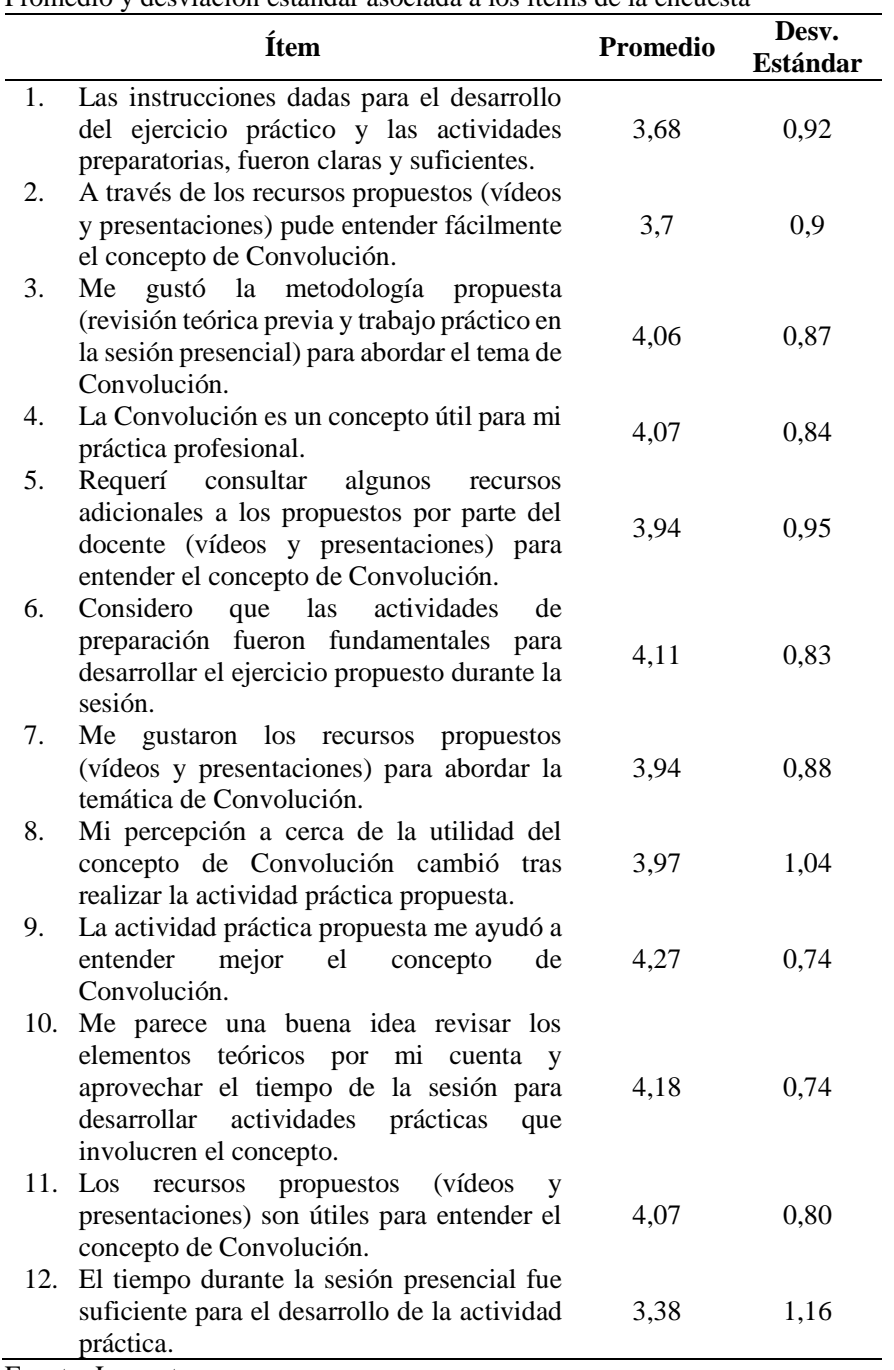

Fuente: Los autores. 
Tabla 2

Promedio y desviación estándar asociada a los ítems de la encuesta agrupados por categoría de análisis

\begin{tabular}{lccc}
\multicolumn{1}{c}{ Categoría } & $\begin{array}{c}\text { Ítems } \\
\text { Categoría }\end{array}$ & Promedio & $\begin{array}{c}\text { Desv. } \\
\text { Estándar }\end{array}$ \\
\hline Recursos educativos & $2,5,7,11$ & 3,91 & 0,88 \\
Metodología & $3,6,10,9,4,8$ & 4,11 & 0,84 \\
Aspectos operativos & 1,12 & 3,53 & 1,04 \\
\hline Fuente: Los autores. & \multicolumn{2}{l}{}
\end{tabular}

fundamentales para desarrollar el ejercicio práctico (ítems 9 y 6). Asimismo, a los estudiantes les gustó la metodología propuesta para abordar el concepto de convolución y les pareció una buena idea revisar los elementos teóricos por su cuenta para aprovechar el tiempo de la sesión desarrollando actividades en las que se aplicaran los conceptos aprendidos (ítems 3 y 10). De otro lado, los estudiantes reportaron una percepción menos positiva en relación al tiempo disponible durante la sesión para el desarrollo de la actividad práctica y a las orientaciones dadas para realizar las actividades preparatorias y la actividad práctica durante la sesión presencial (ítems 1 y 12).

Estos hallazgos son consistentes con los mostrados en la Tabla 2, en la que se presentan los resultados de la encuesta agrupados en las categorías consideradas para el diseño del instrumento: recursos educativos, metodología y aspectos operativos. Como se puede observar en esta tabla, los ítems asociados a la categoría de metodología fueron los mejor evaluados por parte de los estudiantes, mientras que los relacionados con los aspectos operativos presentan los promedios más bajos.

Por otra parte, cabe resaltar que aunque los promedios de los diferentes ítems y categorías (Tablas 1 y 2) muestran una tendencia hacia una percepción positiva respecto a los elementos que integran la nueva estrategia propuesta, los valores de las desviaciones estándar indican variaciones en las percepciones de los diferentes estudiantes que diligenciaron la encuesta.

Con el fin de identificar posibles causas de estas variaciones en las percepciones de los estudiantes y profundizar sobre los hallazgos cuantitativos, se analizaron los comentarios adicionales incluidos por parte de los estudiantes al final de la encuesta. En términos generales, los comentarios realizados se pueden enmarcar en las categorías propuestas para la construcción de la encuesta y posterior análisis de los resultados: recursos educativos, metodología y aspectos operativos.

Una proporción considerable (62\%) de los comentarios realizados en relación a los recursos educativos indican la necesidad de mejorar los recursos propuestos para abordar el concepto de convolución. Estos comentarios hacen alusión principalmente a la dificultad de entender el concepto presentado en un vídeo en inglés y la necesidad de incluir dentro del material de apoyo recursos relacionados con la herramienta de simulación propuesta para desarrollar la actividad práctica. De otro lado, cerca de un 38\% de los comentarios realizados resaltan aspectos positivos en relación a los vídeos y presentaciones sugeridas para abordar el concepto de convolución. En algunos de estos comentarios inclusive se reporta como un acierto la propuesta de incentivar la consulta de recursos en inglés.

Respecto a la metodología propuesta, en la mayoría de comentarios realizados (73\%) se destaca que la nueva estrategia es apropiada para abordar el tema de convolución (favoreciendo una aproximación al concepto a través de una aplicación práctica) y que es conveniente destinar el tiempo de trabajo presencial para profundizar y aplicar los conceptos estudiados previamente por parte de los estudiantes. Adicionalmente, en algunos de estos comentarios los estudiantes resaltan que esta metodología les permite autorregular su proceso de aprendizaje. Por otra parte, en $27 \%$ de los comentarios relacionados con aspectos metodológicos se sugiere ajustar la estructura de la sesión presencial (durante la que se desarrolla la actividad práctica), destinando algunos minutos para resolver inquietudes sobre los elementos teóricos (al inicio de la sesión) y para hacer evidentes las relaciones entre el componente práctico y teórico (al final de la sesión).

Finalmente, en concordancia con los resultados cuantitativos reportados en las Tablas 1 y 2 , todos los comentarios realizados respecto a los aspectos operativos (16 comentarios) reflejan que el tiempo para realizar la actividad práctica no es suficiente y que hace falta claridad en las instrucciones y orientaciones previas al desarrollo de la actividad durante la sesión presencial.

\section{Discusión}

En general, los resultados muestran que la percepción de los estudiantes acerca de las actividades desarrolladas utilizando la metodología de aula invertida es positiva. Esto coincide con resultados reportados en otras experiencias semejantes. Por ejemplo, en el marco del presente estudio se encontró que los estudiantes identificaron algunos componentes de la metodología propuesta como elementos facilitadores para el entendimiento del concepto de convolución (ítem 9 de la Tabla 1), así como en el curso de señales y sistemas de la Universidad de Chalmers, donde los estudiantes expresaron que la metodología les permitió entender mejor los conceptos abordados en esta asignatura [11]. De manera semejante, en el curso de señales y sistemas de la Universidad de Binghamton, los estudiantes reportaron que el proceso de aprendizaje fue "mucho mejor" utilizando la metodología de aula invertida [5].

De otro lado, aunque se evidenció una tendencia positiva en las percepciones de los estudiantes hacia los recursos educativos propuestos para abordar los conceptos y elementos teóricos de manera autónoma en el marco de la metodología del aula invertida (ítems 2, 7 y 11 de la Tabla 1), algunos estudiantes manifestaron dificultades asociadas a la comprensión del concepto a través de los recursos propuestos. Estas dificultades podrían explicarse desde la perspectiva de los estilos de aprendizaje de los estudiantes. En particular, la prevalencia de un determinado estilo de aprendizaje en un estudiante podría dificultar la comprensión de un determinado concepto a través de contenidos o recursos con énfasis en elementos visuales o auditivos. Asimismo, estas dificultades pueden estar relacionadas con el hecho de que algunos estudiantes tienden a preferir explicaciones personales (que 
involucran una interacción directa con el docente) sobre el uso de vídeos [4].

De igual manera, estos elementos se relacionan con procesos de autorregulación de aprendizaje. En algunos casos el aprendizaje autónomo fuera del salón de clases es un desafío para los estudiantes que son los responsables de su aprendizaje [6] y que hasta ahora están familiarizándose con nuevas metodologías de estudio. Los resultados cuantitativos y cualitativos presentados en este artículo muestran que en general los estudiantes consideran apropiado estudiar los elementos teóricos (asociados al concepto de convolución) por su cuenta y aprovechar el tiempo en aula para desarrollar actividades en las que se apliquen y contextualicen estos conceptos. Algunos estudiantes reportaron que esta metodología les permitía avanzar en el proceso de aprendizaje a su propio ritmo, además de considerar más significativo para su aprendizaje complementar este aspecto con el desarrollo de una actividad colaborativa en la que se aplicaba el concepto a una situación real. Este resultado concuerda con lo expresado por los estudiantes del profesor Fowler, quienes reportaron que la atención en clase es mayor con estrategias como la del aula invertida que con una metodología centrada en el docente [5].

Finalmente, los resultados obtenidos permitieron identificar que las variables relacionadas con aspectos operativos pueden llegar a ser determinantes en la implementación de la estrategia propuesta para abordar el concepto de convolución. De esta manera, para próximas implementaciones será necesario proponer diversos tipos de recursos educativos que se puedan ajustar a las preferencias de los diferentes estudiantes, garantizar que las instrucciones y orientaciones definidas para el desarrollo de las actividades son suficientes y claras, sugerir recursos adicionales para introducir aspectos relacionados con la herramienta de simulación propuesta para el desarrollo de la actividad práctica y destinar un tiempo de la sesión presencial para hacer evidente la articulación de los elementos teóricos y la actividad práctica.

\section{Conclusiones}

En el presente estudio se implementó una estrategia de aula invertida para abordar la temática de convolución en el marco de la asignatura de Señales y Sistemas I de la Universidad Nacional de Colombia. La mayoría de estudiantes que participaron en este proceso de implementación consideraron la metodología de aula invertida como una estrategia que les facilitó entender los elementos teóricos y aplicaciones prácticas del concepto de convolución.

Como parte de los resultados generados en esta experiencia, se evidenció que los recursos educativos propuestos para ser abordados de manera autónoma por los estudiantes pueden llegar a ser un factor determinante en la implementación de la estrategia de aula invertida. De esta manera, es importante que los recursos propuestos sean seleccionados considerando (en la medida de lo posible) los diversos estilos de aprendizaje y características de los estudiantes. Aunque es difícil que un sólo recurso educativo sea apropiado para todos los estudiantes, es posible sugerir una serie de recursos que en conjunto posibiliten la comprensión de los conceptos a través de diversas estrategias y mecanismos de instrucción. De igual forma, en el marco de esta experiencia en la que la estrategia activa para la contextualización del concepto de convolución consistía en una actividad práctica desarrollada en una herramienta de simulación, es indispensable sugerir recursos educativos que familiaricen a los estudiantes con el uso de la herramienta.

Asimismo, aspectos operativos relacionados con las orientaciones para el desarrollo del trabajo autónomo por parte de los estudiantes y la actividad práctica en el aula, junto con el tiempo disponible para el desarrollo de la actividad presencial, son factores que incidieron en la percepción de los estudiantes acerca de la implementación de la estrategia de aula invertida. Estas variables, junto con la selección e inclusión de diversos tipos recursos educativos para abordar aspectos teóricos asociados al concepto de convolución y para familiarizar a los estudiantes con la herramienta de simulación propuesta para el desarrollo de la actividad práctica, constituyen algunos de los elementos que deberían considerarse en próximas implementaciones.

\section{Referencias}

[1] Gannod, G., Burge, J. and Helmick, M., Using the inverted classroom to teach software engineering, in Proc. Int. Conf. Softw. Eng., Leipzig, Germany, May 2008, pp. 777-786. DOI: 10.1145/1368088.1368198

[2] Van-Veen, B., Flipping signal-processing instruction. IEEE Signal Processing Mag., 30(6), pp. 145-150, 2013. DOI: 10.1109/MSP.2013.2276650

[3] Mason, G.S., Shuman, T.R. and Cook, K.E., Comparing the effectiveness of an inverted classroom to a traditional classroom in an upper-division engineering course. IEEE Trans. Educ., 56(4), pp. 430435, 2013. DOI: 10.1109/TE.2013.2249066

[4] Bishop, J.L. y Verleger, M.A., The flipped classrom: A survey of the research, 2013 ASEE Annual Conference \& Exposition, Atlanta, Georgia, 2013, pp. 23.1200.1-23.1200.18.

[5] Fowler, M.L., Flipping signals and systems - Course structure \& results, 2014 IEEE International Conference on Acoustics, Speech and Signal Processing (ICASSP), Florence, 2014, pp. 2219-2223. DOI: 10.1109/ICASSP.2014.6853993

[6] Karabulut-Ilgu, A., Jaramillo, N. and Jahren, C.T., A systematic review of research on the flipped learning method in engineering education, British Journal of Educational Technology, 00(00), 2017. DOI: 10.1111/bjet.12548

[7] Ankeny, C.J. and Krause, S.J., Flipped biomedical engineering classroom using pencasts and muddiest point Web-enabled tools, 121st ASEE Annual Conference and Exposition: 360 Degrees of Engineering Education - Indianapolis, IN, United States, 2014, pp. 24.614.124.614.17.

[8] Jungic, V., Kaur, H., Mulholland, J. and Xin, C., On flipping the classroom in a large first year calculus courses, International Journal of Mathematical Education in Science and Technology, 46(4), pp 508-520, 2014. DOI: 10.1080/0020739X.2014.990529

[9] Talbert, R., Learning MATLAB in the inverted classroom, 2012 ASEE Annual Conference \& Exposition, San Antonio, Texas, 2012, pp. 25.883.1-25.883.19.

[10] Buck, J.R. and Wage, K.E., Active and cooperative learning in signal processing courses, IEEE Signal Processing Magazine, 22(2), pp. 76-81, 2005. DOI: 10.1109/MSP.2005.1406489

[11] Svensson, L. and Adawi, T., Designing and evaluating a flipped signals and systems course, Proceedings of the 14th European Conference on eLearning - ECEL 2015, Hatfield, UK, 2015, pp. 584-591. 
A.M. Reyes-Parra, es Ing. Electrónica (2011) y MSc. en Ingeniería Automatización Industrial (2013) de la Universidad Nacional de Colombia. Actualmente es estudiante de doctorado en Ingeniería Eléctrica de la Universidad Nacional de Colombia y docente ocasional del Departamento de Ingeniería Eléctrica y Electrónica de la misma universidad.

ORCID: 0000-0001-9128-8051

M.J. Cañón-Ayala, es Ing. Industrial de la Universidad Central (2012), MSc. en Ingeniería Automatización Industrial de la Universidad Nacional de Colombia (2015) y se ha desempeñado como profesor ocasional del Departamento de Ingeniería Eléctrica y Electrónica de la Universidad Nacional de Colombia.

ORCID: 0000-0002-0181-3156

F.A. Olarte-Dussan, es Ing. Electrónico de la Universidad Distrital Francisco José de Caldas (2005), MSc. en Ingeniería Automatización Industrial de la Universidad Nacional de Colombia (2007) y Dr. en Ingeniería Eléctrica de la Universidad Nacional de Colombia (2011). Actualmente es profesor del Departamento de Ingeniería Eléctrica y Electrónica de la Universidad Nacional de Colombia.

ORCID: 0000-0001-8450-0691 